\title{
Femtosecond laser inscribed superstructure fibre gratings
}

\author{
K. Kalli ${ }^{1 *}$, C. Koutsides ${ }^{1,2}$, E. Davies ${ }^{2}$, M. Komodromos ${ }^{3}$, D. J. Webb ${ }^{2}$, L. Zhang ${ }^{2}$ \\ ${ }^{1}$ Nanophotonics Research Laboratory, Cyprus University of Technology, 3036, Lemessos, Cyprus \\ ${ }^{2}$ Photonics Research Group, Aston University, B4 7ET, Birmingham, United Kingdom \\ ${ }^{3}$ Frederick University Cyprus, Nicosia, Cyprus
}

\begin{abstract}
We demonstrate the development of femtosecond laser inscribed superstructure fiber gratings (fsSFG) in silica optical fibre. We utilise a single step process, to inscribe low loss and polarisation independent, sampled gratings in optical fibres using the point by point femtosecond laser inscription method. Our approach results in a controlled modulated index change with complete suppression of any overlapping LPG structure leading to highly symmetric superstructure spectra, with the grating reflection well within the Fourier design limit. We also solve Maxwell's equations and calculate the back reflection spectrum using the bidirectional beam propagation method (BiBPM). Experimental results validate our numerical analysis and the estimation of inscription parameters such as ac index modulation, wavelength and the relative peak strength. We also explore how changes in the grating's period influence the reflection spectrum.
\end{abstract}

Keywords: Femtosecond laser, superstructure grating, beam propagation method

\section{INTRODUCTION}

The use of femtosecond lasers for the inscription of Bragg and long period gratings has made possible the creation of gratings with special features, and for which their development would be difficult with the conventional UV laser inscription techniques. Fibre Bragg gratings (FBG) have a periodic refractive index modulation of the core with a sub micron pitch, reflecting light at the Bragg resonance wavelength with the forward guided mode coupling to a backward mode and to cladding modes. On the other hand, long period gratings (LPG) have much larger period (few hundred microns) and couple light from the core to forward propagating cladding modes producing broad spectral loss bands as they subsequently couple to radiation modes. These gratings have been systematically investigated and their properties are well known [1], but the inscription of FBG and LPG in the same fibre and the development of superstructure fibre gratings (SFG) is a challenge of its own. From their interesting properties several techniques have been developed for SFG inscription, but these have involved a two-step process that is time consuming and difficult to repeat with poor control over the mean index change and the spectral characteristics [2,3]. We report on the fabrication of SFG exclusively using a femtosecond laser, via a simple, rapid and flexible production process. A key advantage of the approach is that the impact of co-directional cladding mode coupling affecting the Bragg grating reflection response can be effectively eliminated during inscription. This is possible because the femtosecond inscription leads to both positive and negative index changes, leading to approximately zero spatially averaged index change. The gratings can be written with well defined reflectivities and at design wavelengths that precisely coincide with the desired Bragg wavelength. Finally, the femtosecond gratings are of very low loss, displaying uniform spectra; characteristics that are deemed extremely important for a number of applications where it is necessary for the light to penetrate the full grating length and for each of the individual grating elements to contribute to the reflection response.

A femtosecond laser system (HighQ Laser Femtoregen IC 355) emitting $300 \mathrm{fs}$ pulses at $1035 \mathrm{~nm}$ and operating at a repetition rate of $1 \mathrm{kHz}$ was used to inscribe the SFG via the point-by-point method. The laser light was focused with a Mitutoyo microscope objective (x50, NA 0.42 ), which is not very sensitive to fibre curvature effects, allowing for the Bragg structure to be inscribed without oil immersion techniques. The SFG amplitude profile was generated using a mechanical shutter system that enabled us to set the period for the on-off cycles of the SFG. Laser pulses $(3.5 \mu \mathrm{J} / \mathrm{pulse}$ at the laser exit) were attenuated to optimize the laser energy at the sample focus and inscription losses were less than $0.1 \mathrm{~dB} / 5 \mathrm{~mm}$.

*kkalli@cytanet.com.cy

21st International Conference on Optical Fiber Sensors, edited by Wojtek J. Bock, Jacques Albert, Xiaoyi Bao, Proc. of SPIE Vol. 7753, 775393 · (c) 2011 SPIE · CCC code: 0277-786X/11/\$18 · doi: 10.1117/12.885994 
Devices with lengths between 2.2 and $2.5 \mathrm{~cm}$ were written in standard SMF28e optical fibre by scanning the fibre with respect to the femtosecond laser beam. The amplitude period was varied from 100 to 600 microns and the FBG period from 1078 to $1085 \mathrm{~nm}$. Control over the translation speed and laser repetition rate provided control over the pitch of the structure, thereby offering a flexible and fast inscription technique. As the structures were written just above the nonlinear material threshold we maintained good control of the refractive index modulation. The last parameter is of great importance for superstructure grating development as for a weak grating the reflection spectrum is related to the Fourier transform of the refractive index modulation. Another advantage of this approach is that the impact of co-directional cladding mode coupling affecting the grating reflection response can be effectively eliminated during inscription. This is possible because the femtosecond inscription leads to both positive and negative index changes, leading to approximately zero spatially averaged index change as it confirms by inverse Abel transformation [4]. The gratings can be written with well defined reflectivities and at design wavelengths that precisely coincide with the desired Bragg wavelength.

\section{RESULTS}

The transmission and reflection spectra were measured using a broadband light source and an optical spectrum analyzer. The laser inscription energy was kept just above the damage threshold for the germanium doped fibre core, from which phase contrast optical measurements and inverse Abel transforms indicate an ac index change of $8 \times 10^{-4}$. The mean dc index change was $3.16 \times 10^{-5}$ and depends critically on the pulse energy, as does the minimisation of device loss and polarization dependence $[4,5]$. UV inscribed gratings typically have mean index changes an order of magnitude greater. The grating strength is expressly kept to $1.2 \mathrm{~dB}$ or less as this falls within the Fourier design limit that demands that gratings have a reflectivity of less than $25 \%$. Fig. 1(a) shows both a transmission spectrum for a typical LPG accompanied by one for a SFG, both devices have an amplitude modulation period of 600 microns, but in the latter case the laser energy is reduced by $5 \%$, which proves sufficient to completely suppress the LPG response. However the laser pulse energy remains just above the material-dependent threshold to allow for the structured FBG. Fig. 1(b) shows the transmission and reflection response for a SFG having a Bragg period of $1078 \mathrm{~nm}$, while the amplitude period is 300 microns. There is no evidence of the LPG response, but we observe the characteristic Bragg cladding mode resonances.
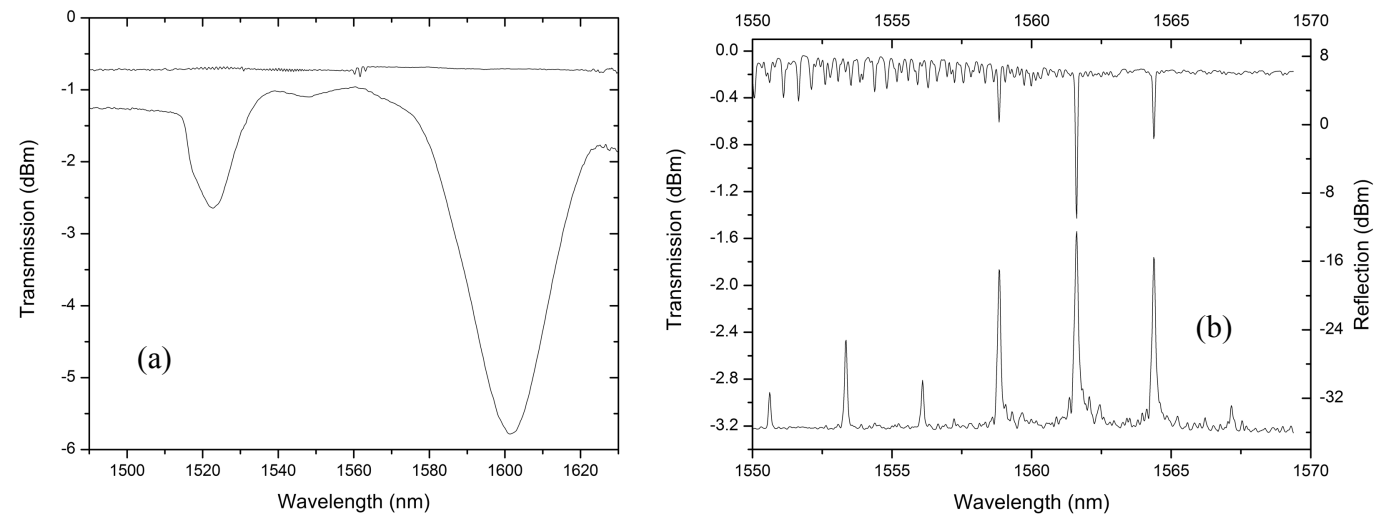

Fig.1. (a) Transmission spectrum for LPG and SFG (with LPG completely suppressed) for amplitude modulation period of $600 \mu \mathrm{m}$, (b) SFG transmission and reflection response for a Bragg period of $1078 \mathrm{~nm}$, and amplitude period of $300 \mu \mathrm{m}$.

Fig. 2 shows the characteristic reflection spectra for three SFG with different FBG period but equal "LPG" period of 600 $\mu \mathrm{m}$; a red shift appears as the FBG's period increases. We use the bidirectional beam propagation method (BiBPM) in order to solve Maxwell's equation and to calculate the reflection spectra. As we observe from Fig. 2 our analysis confirms that a difference in the FBG period leads to a spectral red shift, showing that the numerically computed reflected spectra for $1078 \mathrm{~nm}, 1082 \mathrm{~nm}$ and $1085 \mathrm{~nm}$ FBG periods are in excellent agreement with experimentally measured spectra, whilst predicting almost all the experimentally measured resonances. Furthermore, the peaks evaluated for different values of the FBG period with same period of refractive index or LPG modification show that the FBG period determines the resonance wavelength of the central peak; shifting the power envelope distribution without affecting the phase of the sinc function. 

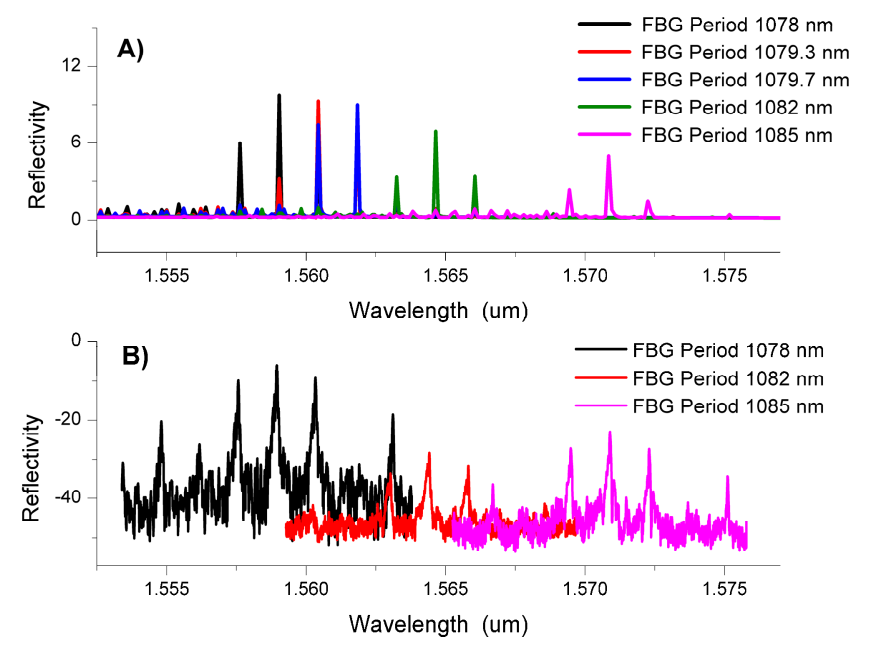

Fig. 2 (a) Numerical modelling of the SFG reflection spectra for different FBG periods, (b) equivalent experimental reflection spectra

Furthermore, for a given LPG spacing, the reflection peaks for any given FBG period precisely overlap with peaks from any another FBG period. The experimental results show and numerical results predict that as the FBG period changes there are discrete spectral "steps" and all resonance peaks overlap. Of course, the distance between the central resonance and the side band peaks predominantly depends on the LPG or refractive index modulation and is consistent with Equation (1) that predicts that side band peaks spacing mainly influence by LPG period

$$
\Delta \lambda_{s} \approx \frac{\lambda_{\mathrm{B}}^{2}}{2 n_{\text {eff }} \Lambda_{L P G}}
$$

where $\Delta \lambda_{\mathrm{s}}$ is the wavelength separation between central peak and side band peaks, $\lambda_{\mathrm{B}}$ is the Bragg resonance wavelength and $\Lambda_{\text {LPG }}$ is the spatial shift for the LPG. Fig. 3 summarizes these results and shows numerical analysis for four gratings' resonance wavelength as a function of their FBG and LPG period. The FBG period is six orders greater than the LPG period in determining the peak resonance wavelength, clearly impacting the measurement of small axial strains.
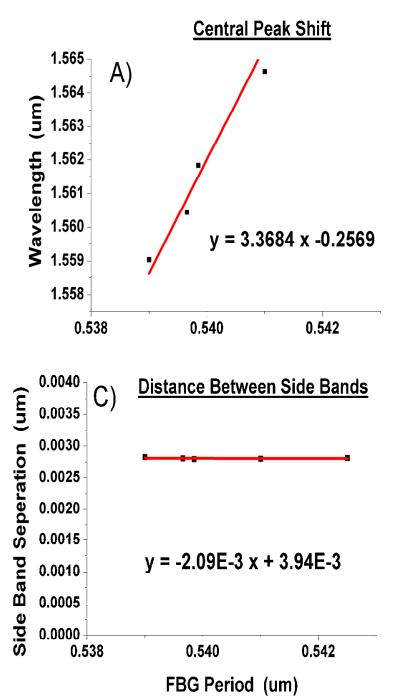

Fig. 3 (a) Central peak resonance wavelength with FBG period, (b) Central Peak wavelength with LPG period, Side band separation with (c) FBG period and (d) with LPG Period
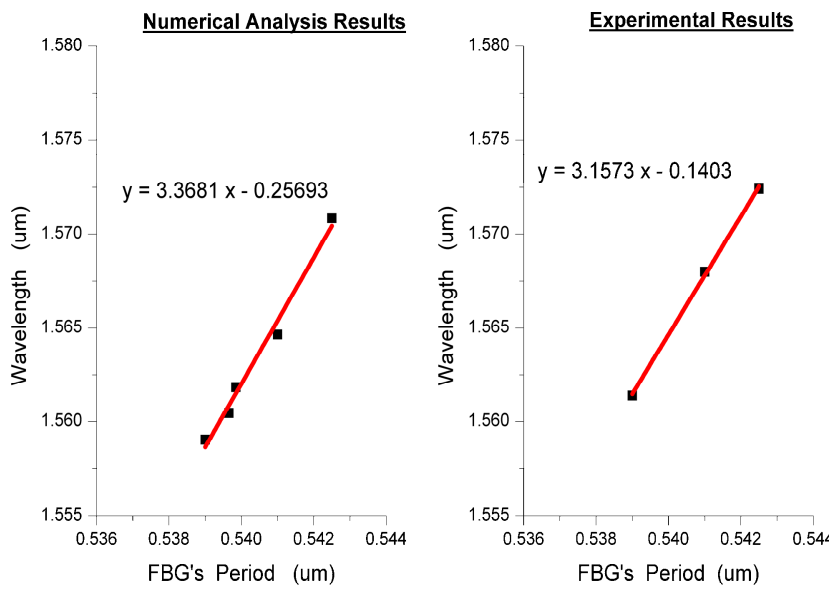

Fig. 4 Calculated and experimental results for the resonance wavelength according to the FBG period 
On the other hand, as the spatial separation of the FBG increases (lower refractive index modulation frequency); side band resonances approach the central wavelength. The negative sign in the linear equation in Fig. 3(d) predicts that relationship while Fig. 3(c) shows that side band distance is not affected by changes in the FBG period. In order to support our theoretical results we favourably compare calculated results with experimentally measured spectral shifts proportional to the change in the FBG period, Fig. 4. The linear equations that describe the red shift in the experimental measurements and numerical analysis are very similar with a slope deviation below $6 \%$, which is acceptable if we consider the complexity of such structures and that even sub micron lateral shifts of the grating from fibre's propagation axis can introduce changes in the transmission signal because of symmetry breaking of the structure. Furthermore, any possible errors in the LPG and FBG measurements in the inscribed structures will introduce deviations in the results.

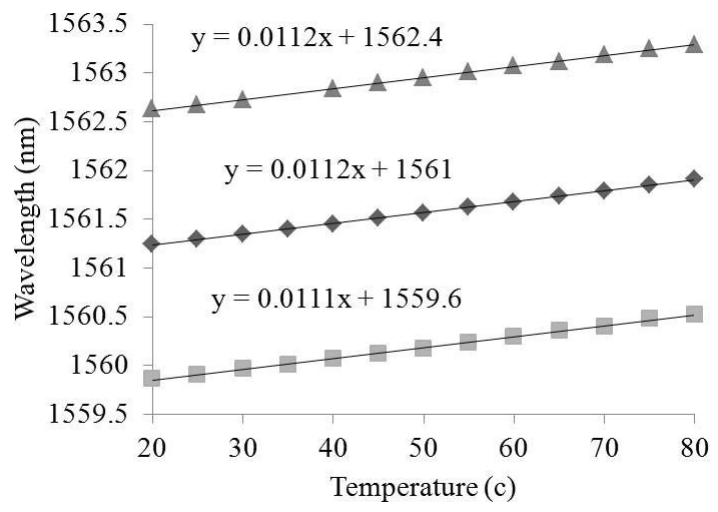

Fig. 5 The measured temperature response of the three strongest reflection peaks of a typical SFG device

Finally, in Fig. 5 we observe the measured temperature response of the three strongest reflection peaks of a typical fsSFG device, producing a wavelength to temperature response typical for a $\mathrm{FBG}$ based device of $11.2 \mathrm{pm} /{ }^{\circ} \mathrm{C}$, for each peak.

\section{CONCLUSIONS}

We present a rapid and flexible method to produce SFG using the femtosecond laser point-by-point technique. The flexibility to alter the grating parameters, allowing for variations in the grating length, pitch, and spectral response are incorporated easily. Control of the laser pulse energy can effectively suppress any LPG response; thereby avoiding the typical two step process for UV inscribed SFG. We note that even minute changes in FBG leads to a red shift to the reflection spectrum without affecting power distribution of the resonance peaks, which is modified by the refractive index or "LPG" profile. We have successfully modelled experimental results using the BiBPM. Finally, the femtosecond gratings are of very low loss, displaying uniform spectra; characteristics that are deemed extremely important for a number of applications where it is necessary for the light to penetrate the full grating length and for each of the individual grating elements to contribute to the reflection response.

\section{REFERENCES}

[1] Othonos A., and Kalli K. 'Fibre Bragg gratings - fundamentals and applications in telecommunications and sensing'. (Artech House, Boston, 1999)

[2] Eggleton B. J., Krug P. A., Poladian L., and Ouellette F. 'Long periodic superstructure Bragg gratings in optical fibres', Electron. Lett., 30, 1620 - 1622 (1994)

[3] Broderick N. G. R., and De Sterke C. M. 'Theory of grating superstructures', Phys. Review E, 55, 3634-3646 (1997)

[4] Kalli K., Allsop T., Koutsides C., Davies E., Webb D., and Zhang L. 'Femtosecond laser inscription of fibre Bragg gratings with low insertion loss and minor polarization dependence', in Bragg Gratings, Photosensitivity, and Poling in Glass Waveguides, OSA Technical Digest (Optical Society of America, 2010), paper BMA3.

[5] Geernaert T., Kalli K., Koutsides C., Komodromos M., Nasilowski T., Urbanczyk W., Wojcik J., Berghmans F., and Thienpont H.: 'Point-by-point fibre Bragg grating inscription in free-standing step-index and photonic crystal fibres using near-IR femtosecond laser', Opt. Lett., 35, 1647-1649 (2010) 